\title{
Visual parsing and response competition: The effect of grouping factors
}

\author{
GORDON C. BAYLIS \\ University of California, San Diego, California \\ and \\ JON DRIVER \\ University of Cambridge, Cambridge, England
}

\begin{abstract}
When the task is to categorize a target letter at a known location, subjects show more interference from incongruent distractors that are relatively close (B. A. Eriksen \& C. W. Eriksen, 1974) or that share common motion with the target (Driver \& Baylis, 1989). In eight experiments, we examined whether static factors other than proximity can affect the amount of interference. When distractors and the target letter were in the same color, the distractors interfered more than they did when they were in a different color, even when the latter were closer to the target. Good continuation between the target and distractors also led to more interference. These results suggest that the efficiency of selection is determined by several aspects of the relation between targets and distractors in addition to their proximity, and thus that visual attention is not directed on the basis of position information alone.
\end{abstract}

The selective mechanisms of visual attention allow us to respond to individual objects in cluttered visual scenes. A popular conception of these processes is that their operation is analogous to a spotlight "illuminating" or a zoom lens "magnifying" a particular region of the visual field to allow a more precise coding of events in that region (see, e.g., Broadbent, 1982; B. A. Eriksen \& C. W. Eriksen, 1974; C. W. Eriksen \& St. James, 1986; La Berge, 1983; Posner, 1980; Tsal, 1983). The classic findings of B. A. Eriksen and C. W. Eriksen (1974) were influential in the development of this view. Their subjects made a two-choice response to a letter appearing at a known locus. Distracting letters associated with the alternative response slowed this reaction, producing response competition. However, the interference from distractors was only found when they were close to the target; beyond about $1^{\circ}$, they had little or no effect. This distance effect has been replicated many times, although there is some controversy over the existence of a critical separation beyond which interference from distractors is completely eliminated (Miller, 1991). The interpretation of the distance effect in terms of spotlight or zoom lens theory is

This research was supported by US Office of Naval Research Contract N00014-88-K-0281 to G. C. Baylis. J. Driver was supported by a Fellowship at the McDonnell-Pew Center for the Cognitive Neuroscience of Attention, University of Oregon. The authors would like to thank Zoltan Dienes and Ethan Newby for running subjects in Experiments 2, 4, 5, and 6. We are grateful to Charles Eriksen, Art Kramer, Tram Neill and an anonymous reviewer for their many constructive criticisms of an earlier version of the manuscript. Correspondence concerning this article should be addressed to G. C. Baylis, Department of Psychology 0109, University of California, San Diego, La Jolla, CA 92093. as follows. Attention is focused on the known target location. Distractors close to this focus will be processed more fully than will distractors that are farther away, since only the close distractors fall within the "illuminated" or "magnified" region. Hence, near distractors produce more response competition than do far distractors, as B. A. Eriksen and C. W. Eriksen (1974) found.

Driver and Baylis (1989) examined whether proximity to the target was the only factor that would determine the amount of response competition produced by incongruent distractors. They found that distant distractors that moved with a target could produce more interference than did distractors that were closer to the target but did not share its motion. They interpreted this to mean that the grouping of target and distractors by common fate (Wertheimer, 1922,1923 ) overrode the effects of grouping by proximity. The implication is that rather than being directed to unparsed regions of space, attention is applied to perceptual groups, as Duncan (1984), Kahneman and Henik (1981), and Prinzmetal (1981) have suggested. If so, the special status afforded to position by literal versions of the spotlight and zoom lens metaphors may not be justified. Instead, proximity may be just one of many grouping factors (albeit a very powerful one), as the Gestaltists proposed.

Driver and Baylis (1989) did not implement any formal measure of phenomenal grouping, so that to describe their result in these terms requires extrapolation from previous studies on the role of motion in phenomenal grouping (e.g., Wertheimer, 1923). An alternative account of their results that does not rely on such extrapolation is the following. The problem faced by the subject in the Eriksen response-competition paradigm is to pick 
out the target from the distractors and to respond to the identity of the former alone. Near distractors may produce more interference than fae distractors do (B. A. Eriksen \& C. W. Eriksen, 1974), simply because they resemble the target more closely on the attribute used to pick out the target (i.e., location) and are therefore harder to select against. According to this "similarity" view (see Duncan \& Humphreys, 1989), the observations of Driver and Baylis (1989) would be taken to show that motion information can be used in addition to position information in the process of selecting the target. Distractors that resemble the target in motion lead to more interference, just as do distractors that resemble the target in position. Although this account does not invoke phenomenal grouping, for the present purposes it makes the same point as Driver and Baylis's (1989) grouping account-namely, that response competition is not determined by proximity alone, which implies that attention is not directed solely on the basis of position information (cf. Tsal \& Lavie, 1988).

Moving displays such as those employed by Driver and Baylis (1989) may constitute a special case for visual attention, as suggested by Humphreys and Bruce (1989, pp. 167 and 184). When motion is introduced in a display, positions constantly change. As a result, position in moving displays may have less importance than does position in static displays. To investigate this question further, we conducted eight experiments to examine whether response competition can be affected by static visual properties other than proximity (see C. W. Eriksen \& Schulz, 1979, and O'Hara \& Eriksen, 1979, for evidence that encourages this view). Specifically, we examined the effects of color and good continuation. If attention is directed in static displays on the basis of position information alone (as claimed by Tsal \& Lavie, 1988, for example), the amount of response competition from incongruent distractors should depend only on their proximity to the target. By contrast, according to both the grouping and the similarity accounts outlined above, a number of nonspatial properties might exert an effect.

Demonstrations that factors such as motion can sometimes override proximity (e.g., Driver \& Baylis, 1989) are particularly striking, given the well-established effect of proximity (see, e.g., B. A. Eriksen \& C. W. Eriksen, 1974; Miller, 1991). However, it is not necessary to override proximity in order to establish that factors other than proximity (color, for example) can affect response competition. All that would be required would be an effect of color while other possible factors (such as target-distractor separation) are held constant. Since the effect of targetdistractor separation is considerable (see, e.g., B. A. Eriksen \& C. W. Eriksen, 1974), requiring color to override proximity could lead to the premature conclusion that color has no effect, when in fact its effects are simply less powerful than those of proximity for the particular parameters used. A more neutral experimental strategy would begin by seeking an effect of color at a constant target-distractor separation. If such an effect were to become established, one might then investigate whether there were any parameters for which color could override proximity. In our first experiment, we examined whether incongruent letters at a constant distance from a target letter would produce more response competition when they shared the target's color than when they had a different color.

\section{EXPERIMENT 1}

The task was to categorize the central letter in an $X$-shaped array of five letters. The four distractors were equidistant from the central target letter. The two distractor letters along one diagonal had the same color as the target's (red or green), whereas those along the other diagonal were in the other color (see Figure 1a for an example). If response competition in static displays is only affected by target-distractor proximity, incongruent distractors in either diagonal should produce the same amount of interference. On the other hand, if color plays a role analogous to that of proximity, distractors that are grouped with the target (alternatively, which resemble it) via common color should produce more interference.

\section{Method}

Subjects. The 18 subjects, 8 male and 10 female, were lower division psychology undergraduates at the University of California, San Diego. All had normal or corrected-to-normal acuity and normal color vision by self-report. They received course credit for

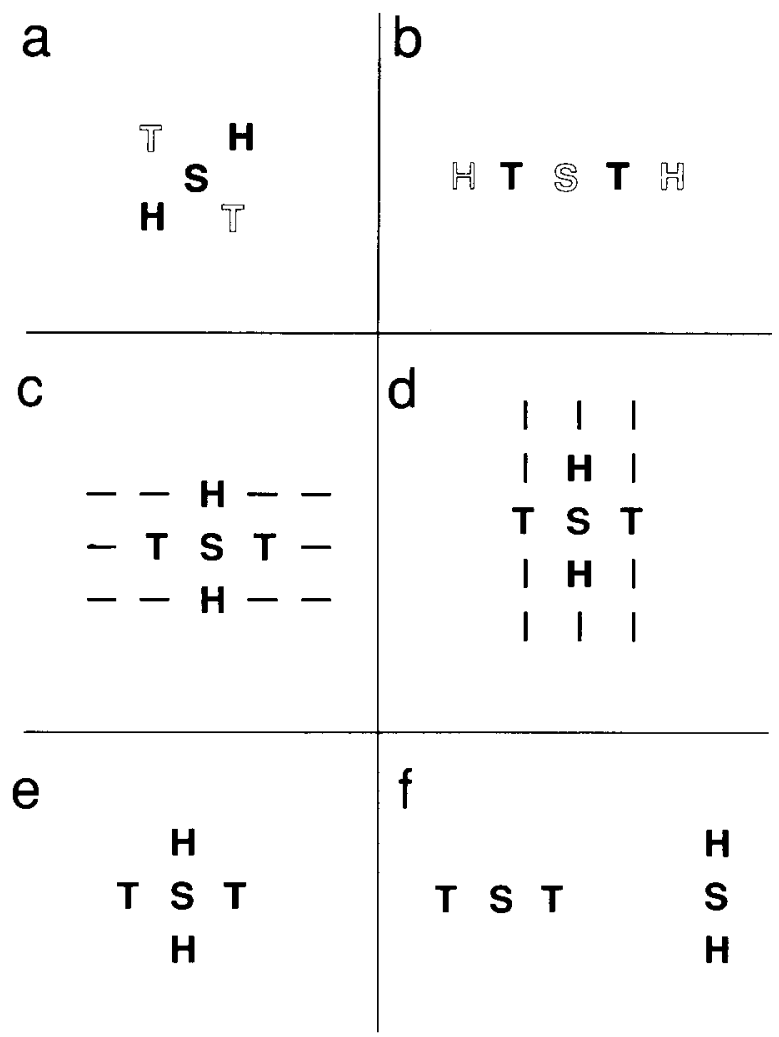

Figure 1. Typical neutral displays from the various experiments. (a) Experiment 1, in which the open type and bold type represent different display colors. (b) Experiment 2, in which the open type and bold type again represent different display colors. (c) Experiment 5. (d) Experiment 6. (e) Experiment 7. (f) Experiment 8. 
participation. The subjects were randomly assigned to two groups of 9 each.

Apparatus and Materials. The experiment was conducted on either a Zenith 286 or a Compaq $386 / 20$ microcomputer. Stimuli were presented in a "stroked" font in graphics mode, enabling accurate placement of stimuli within the display field. EGA doublepaged graphics mode was used to ensure that the onset or the offset of stimuli occurred within a single frame. Displays were presented on a color EGA monitor (Samsung). The uppercase letters $X, Y$, $\mathrm{S}, \mathrm{C}, \mathrm{H}$, and $\mathrm{T}$ were employed. A response was made by pressing one of two keys (" $Z$ " or " $/$ " on the standard extended keyboard) with the left or right index finger, and responses were timed in milliseconds. The diagonal letters $\mathrm{X}$ and $\mathrm{Y}$ were assigned to one response, and the curved letters $S$ and $C$ to the other, the particular hand being counterbalanced across subjects. $H$ and $T$ appeared only as response-irrelevant distractors and were intended to be neutral as regards response tendencies. At the viewing distance of $70 \mathrm{~cm}$, each letter subtended about $0.9^{\circ}$ in height $\times 0.5^{\circ}$ in width.

Each display consisted of five letters: a target letter appearing at fixation surrounded by four distractors in an $\mathrm{X}$-shaped format (see Figure 1a). Each diagonal of three letters was $2.4^{\circ}$ long. The letters were presented in red or green on a black background. For the 9 subjects in the red group, the target was always red, whereas for the 9 subjects in the green group, the target was always green. The two distractors along one diagonal shared the target's color, and those along the other diagonal had the other color. The two distractors along a diagonal were always repetitions of the same letter within a display. There were no repetitions of distractor identity across diagonals within a display.

Design. A mixed design was employed. The between-subject factor was color group (red vs. green target), and the within-subject factor was the relationship of the distractors to the target, leading to five conditions:

Baseline. All items in the display except the target were neutral (i.e., $\mathbf{H}$ or $\mathbf{T}$ ).

Congruent, same color. Distractors in the diagonal that shared the target color were associated with the same response as was the target (e.g., they were Xs if the target was a Y); distractors in the other diagonal were neutral (i.e., Hs or Ts).

Congruent, different color. Distractors in the diagonal sharing the target color were neutral; distractors in the other diagonal were congruent with the target.

Incongruent, same color. Distractors in the diagonal with the target color were associated with the currently incorrect response (e.g., they were Ss or Cs if the target was a Y); those in the other diagonal were neutral.

Incongruent, different color. Distractors in the diagonal with the target color were neutral; those in the other diagonal were incongruent with the target.

Procedure. The subjects were instructed to press the button appropriate to the target letter appearing at fixation, as quickly and accurately as possible. The sequence of events was as follows for each trial: (1) A fixation cross was presented for $500 \mathrm{msec}$; (2) the target and distractors were presented concurrently for $180 \mathrm{msec}$; (3) the subjects responded; and (4) an interval of $800 \mathrm{msec}$ elapsed before the next fixation cross appeared. The subjects were told that they would see five colored letters arranged in an X format, and that they should respond only to the center letter and ignore the flanking distractors. They were presented with six blocks of 150 trials each. Each of the five conditions appeared four times every 20 trials, in a different pseudorandom order for each subject. At the end of each block, the subjects were told their mean reaction times (RT) for correct responses, and their mean error rates. To ensure that they were responding quickly but accurately, the computer displayed a message requesting that they be more accurate in the next block if their error rate had exceeded $15 \%$, and a message telling them to respond more quickly if their error rate had been below $5 \%$. Feedback messages of this type were similarly displayed in Experiments 3-8 below.

Treatment of results. The first 100 trials were discarded as practice, as were the first 2 trials of each block. Thus 790 trials were available for each subject. All these contributed to the accuracy analyses. However, the data were trimmed for RT analyses as follows. Error trials were excluded, as were trials immediately following an error because of the variability they typically introduce (Rabbitt, 1966). It is conventional to exclude RTs beyond some arbitrary minimum and maximum, on the grounds that very fast or very slow responses are likely to arise from different processes than are the responses of interest (e.g., guesses arising because the subject blinked during the display). Following Driver and Baylis (1991), we adopt a principled a priori criterion for determining the cutoffs for acceptable RTs. Upper and lower cutoffs were calculated individually for each subject to remove trials with extremely short or extremely long RTs beyond which accuracy was at chance (i.e., responses were not different from chance as tested by a chi-square test at the .05 level). For example, an individual's upper cutoff was derived by taking all the RT data for that subject sorted by magnitude, selecting the longest RT, then the next longest, and so on, until accuracy on the selected trials exceeded chance. All RTs longer than the final RT selected were then excluded. An analogous process was applied to derive the lower RT cutoffs, working from the opposite tail of the RT distribution.

The combination of upper and lower criteria excluded $3.5 \%$ of the trials. The pattern of results would be the same if these trials were included. This is also the case for all of the subsequent experiments in this paper in which the cutoff treatment was applied, and accordingly, it will not be restated. All statistical analyses were carried out with the use of SYSTAT (Wilkinson, 1986).

\section{Results and Discussion}

The means of subjects' median reaction times for the five within-subject conditions are shown in Figure 2, together with the associated error rates, pooled across the two groups of subjects. A two-way mixed analysis of variance (ANOVA) of the RT data showed a significant effect of within-subject condition $[F(4,64)=18.7, p<.001$, mean square error $(M S E)=133.1]$ and no main effect or interaction involving group $[F(1,16)=1.1$, and $F(4,64)$ $=.6$, respectively]. In subsequent analyses, therefore, data from the two groups were combined. Pairwise comparisons were made here and throughout this paper by means of Neuman-Keuls tests (applying a two-tailed model), in this case following a one-way within-subject ANOVA on the pooled data. With an $M S E$ of 130.5 , this revealed the following pattern of pairwise differences among the conditions. The incongruent same-color condition had slower RTs than did the baseline $(p<.001)$ or incongruent different-color conditions $(p<.01)$. The baseline and incongruent different-color conditions did not differ significantly. Thus, response competition was observed from incongruent distractors in the same color as the target's but not from incongruent distractors that were at the same distance from the target but had a different color.

In agreement with the pattern of results found by Driver and Baylis (1989), neither congruent condition differed significantly from the baseline, and the two congruent con- 

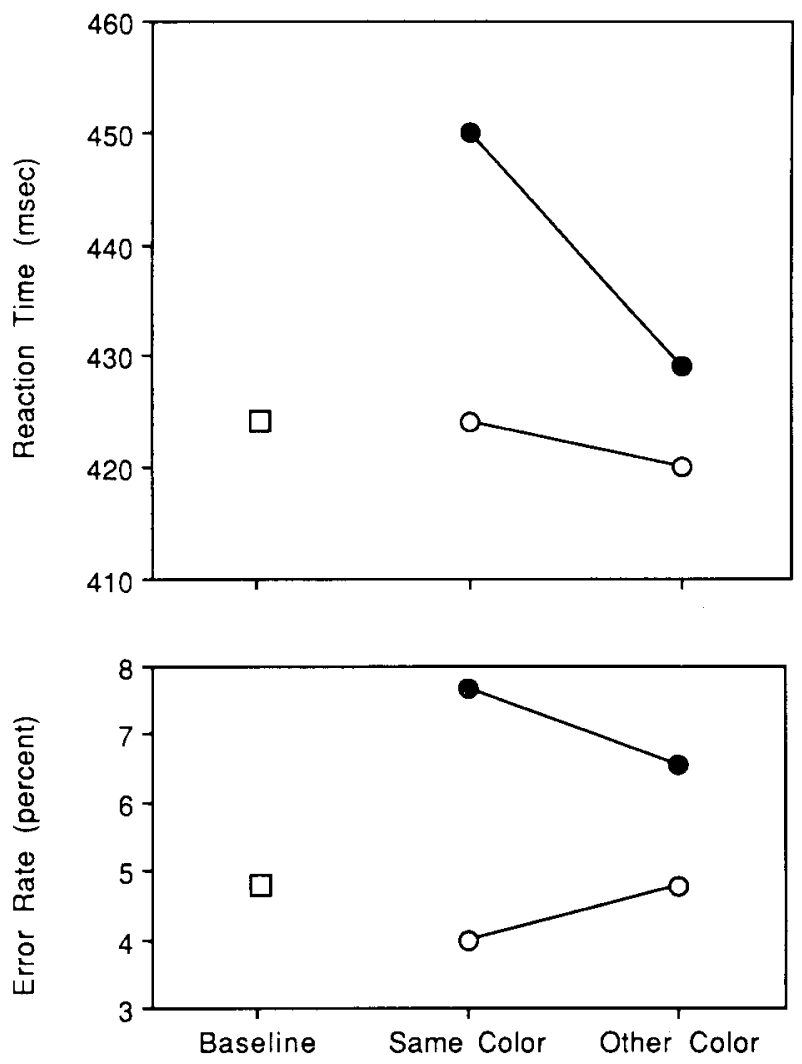

Condition

Figure 2. Mean reaction time (above) and error rate (below) for Experiment 1. Congruent conditions are shown in open circles, incongruent in filled circles, baseline as an open square.

ditions did not differ from each other. These findings also apply for all subsequent experiments in this paper, and for ease of exposition, they will not be restated.

A similar pattern of results was found in the error data. A two-way mixed ANOVA again showed a main effect of condition $[F(4,64)=10.4, p<.001, M S E=3.9]$ with no main effect or interaction involving group $[F(1,16)$ $=.2$ and $F(4,64)=2.0$, respectively, $M S E=28.2$ and 3.9]. Neuman-Keuls comparisons on the pooled data $(M S E=4.18)$ showed that there were more errors in the incongruent same-color and incongruent different-color conditions than in the baseline condition $(p<.001$ and $p<.02$, respectively). The difference between the incongruent same-color and the incongruent different-color conditions approached significance $(p=.08)$. As with the RT data, there were no significant differences between the congruent conditions, or between either of the congruent conditions and the baseline condition. This was also true of the error data from all subsequent experiments in this paper.

The RT data cannot therefore be attributed to any speedaccuracy tradeoffs. Thus, incongruent distractors in the same color as the target's produced more interference than did distractors of a different color that were at the same distance from the target. Proximity is therefore not the only factor to affect the extent of response competition in static displays (cf. Miller, 1991), which suggests that visual attention is not directed on the basis of position alone, even when target location is known in advance (cf. Tsal \& Lavie, 1988). Target-distractor grouping (or similarity) due to common color can have effects similar to those of target-distractor proximity in modulating the extent of response competition. In our second experiment, we examined whether there are any parameters for which the effects of color can override those of proximity.

\section{EXPERIMENT 2}

In this study, we investigated whether common color could produce effects on response competition that were analogous to the effects of common motion observed by Driver and Baylis (1989). If so, distant distractors that share the target's color might under some circumstances produce more interference than would distractors that are closer to the target but that have a different color. The task was to categorize the central letter in a horizontal array of five letters. This central letter had the same color as did the outer letters, whereas the intermediate letters had a different color. We could therefore compare the interference produced by distractors close to the target but in a different color with the interference from distractors farther away that shared the target's color. If visual attention is directed on the basis of position information alone (as suggested by Tsal \& Lavie, 1988), there should be more interference from the closer distractors, regardless of color. However, according to the grouping or similarity accounts, common color could in principle override proximity, depending on the parameters used. If so, the distant distractors would produce more interference as a result of sharing their color with the target. The horizontal array of five letters was centered at fixation, so the distance of distractors from the target was confounded with their distance from the fovea (Hagenaar \& van der Heijden, 1986). This possible artifact would favor more interference from the near than from the far distractors, because the former should have a slight acuity advantage.

\section{Method}

Subjects. The 20 subjects ( 9 male, 11 female), who were 18-45 years old, were paid volunteers from the Oxford University subject panel. All had normal or corrected-to-normal acuity and normal color vision by self-report. They each attended two sessions. The sessions took place on consecutive days, and each session lasted approximately $1 \mathrm{~h}$.

Apparatus and Materials. The apparatus and materials were the same as in Driver and Baylis (1989). The letter stimuli were generated on an Acorn BBC microcomputer (Model B+) with 52K of RAM and presented on a Microvitec Cub 895 Monitor. The uppercase letters X, Y, S, C, H, and T were employed, as in Experiment 1 . A response was made by pressing one of two keys with the right or left index finger, and responses were timed in milliseconds. As before, the diagonal letters $X$ and $Y$ were assigned to 
one response, and the curved letters $S$ and $C$ to the other, the particular hand being counterbalanced across subjects. $H$ and $T$ appeared only as neutral distractors. At the viewing distance of $90 \mathrm{~cm}$, each letter subtended about $.25^{\circ}$ horizontally and $.38^{\circ}$ vertically.

Each display consisted of a row of five letters: a central target immediately flanked by two near distractors in a different color and then by two far distractors in the target's color (see Figure lb). The five letters were horizontally aligned and equally spaced, with the target appearing at fixation. The center-to-center distance between adjacent letters was $.6^{\circ}$, with a minimum $.35^{\circ}$ edge to edge. The fixation cross subtended $.25^{\circ}$ square.

Design. A mixed design was employed. The within-subject factor was the identity and location of target and distractors, leading to five conditions.

Baseline. All items in the display except the target were neutral items (e.g., H T Y TH).

Near congruent. Items in the positions immediately adjacent to the target were associated with the same response as was the target; items in the far positions were neutral (e.g., HXYXH).

Far congruent. Items close to the target were neutral; items in the farther positions were associated with the same response as was the target (e.g., X H Y HX).

Near incongruent. Items in the positions immediately adjacent to the target were associated with the response opposite to the target's; items in the far positions were neutral (e.g., HSYSH).

Far incongruent. Items close to the target were neutral items in the farther positions were associated with the response opposite to the target's (e.g., STYTS).

The between-subject factor was the color of the targets and distractors. For the 10 subjects in the purple group, the target and far distractors were purple, while the near distractors were green; the reverse applied for the $\mathbf{1 0}$ subjects in the green group. Purple and green were chosen because they seemed to the experimenters to be the optimal pair of colors available on the $\mathrm{BBC}$ microcomputer in terms of maximizing discriminability while retaining comparable saliency.

Procedure. The subjects were instructed to press the button appropriate to the target letter appearing at fixation as quickly and accurately as possible. The sequence of events for each trial was the following: (1) The subject pressed a foot-pedal to initiate the trial; (2) the fixation cross was presented for $500 \mathrm{msec}$; and (3) the target and distractors were presented concurrently for $200 \mathrm{msec}$. If the subjects pressed the correct button in response to the display, their RT was displayed on the monitor. If they pressed the inappropriate key, the word "wrong" appeared. Provided that subjects kept the foot-pedal depressed, the feedback message lasted $800 \mathrm{msec}$; it was then replaced by a fixation cross, with the sequence of events repeated to generate the next trial. If they released the foot-pedal during the first $800 \mathrm{msec}$ of feedback, the message remained on the screen for $2 \mathrm{sec}$ and was then replaced by the message "press pedal for next trial." In general, subjects kept the footpedal depressed throughout the experiment unless they stopped to cough or sneeze. Thus, the experiment was primarily forced-paced rather than self-paced. The messages and feedback were presented in the target color (i.e., purple or green) for each subject.

Each subject underwent a total of 12 blocks of 100 trials, 6 on Day 1 and 6 on Day 2. The first 2 blocks on Day 1 were discarded a priori as practice. In addition, error trials and trials following an error were discarded from RT analyses, together with the first two trials in each block. Upper and lower RT cutoffs were not applied, since our cutoff software was not available for the BBC microcomputer. Within each block, the five conditions were equiprobable, as were the four possible target identities. The letters $X, Y, S$, and $\mathrm{C}$ appeared equally often as near or far distractors, as did $\mathbf{H}$ and $T$. Within these constraints, a different random sequence of trials was generated for each subject.

\section{Results and Discussion}

The means of individuals' median RTs, together with the associated error rates, are shown in Figure 3 for each of the five within-subject conditions pooled across the two groups of subjects. A two-way mixed ANOVA of the RT data showed a significant effect of condition $[F(4,72)=$ $9.9, p<.001, M S E=241]$ and no main effect or interaction involving group $[F(1,18)=.02$, and $F(4,72)=$ .7 , respectively, $M S E=15,410.3$ and 241$]$. In subsequent analyses, therefore, data from the two groups were combined. Neuman-Keuls tests (with $M S E$ from the combined data of 236.7) showed that both incongruent conditions were slower than the baseline $(p<.01$ for the far incongruent; $p<.02$ for the near incongruent), while the far incongruent was slower than the near incongruent $(p<.01)$.

A similar pattern of results was found in the error data. A two-way ANOVA again showed a main effect of condition $[F(4,72)=16.2, p<.001, M S E=6.5]$, with no main effect or interaction involving group $[F(1,18)=.79$ and $F(4,72)=.29$, respectively, $M S E=31.5$ and 6.5]. Neuman-Keuls tests $(M S E=6.24)$ showed that the far
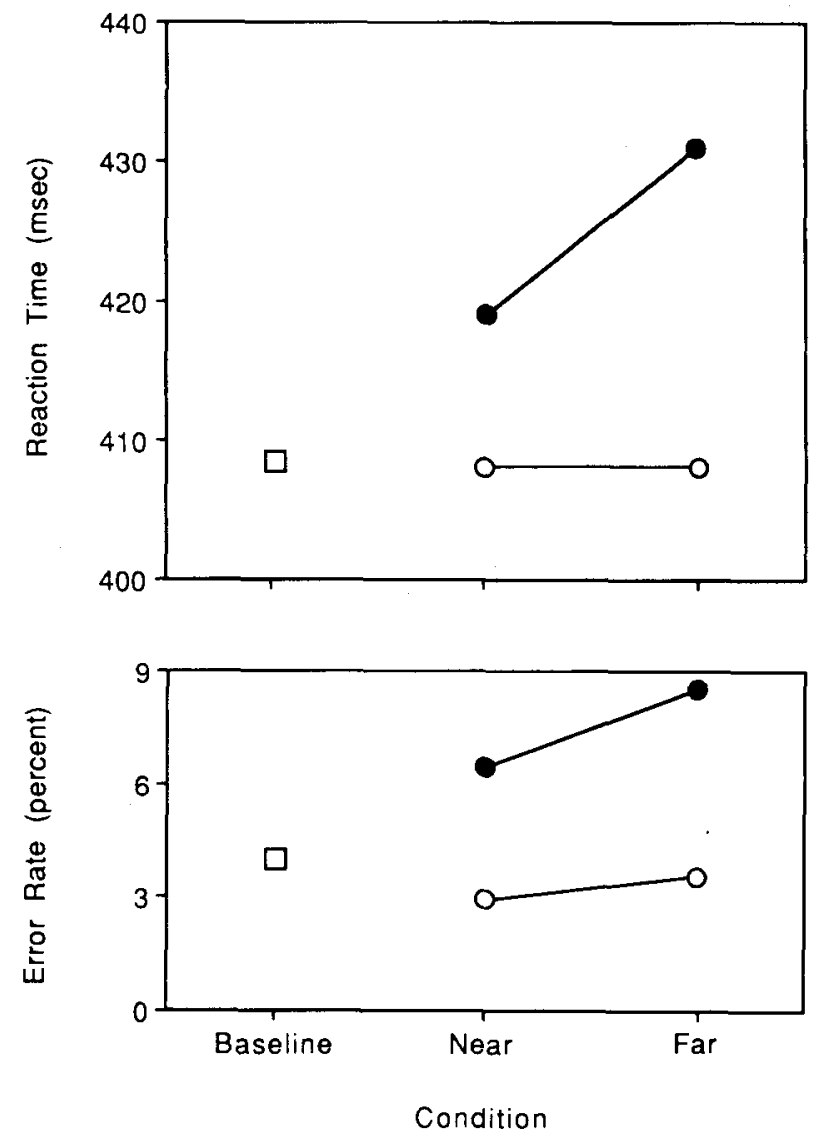

Figure 3. Mean reaction time (above) and error rate (below) for Experiment 2. Congruent conditions are shown in open circles, incongruent in filled circles, baseline as an open square. 
incongruent condition produced more errors than did the near incongruent or baseline $(p<.01)$, while the near incongruent and baseline-also differed $(p<.02)$.

These results suggest that with appropriate parameters (i.e., highly discriminable colors and modest targetdistractor separations), common color can override proximity so that relatively distant distractors with the target's color can produce more response competition than distractors that are closer but have a different color. This finding cannot be due to the far distractors' appearing in a color more salient than that of the near distractors, because the same pattern of results was found (1) when the target and the far distractors were both green and the near distractors were purple and (2) when these colors were reversed.

It might be suggested that the far distractors produced the greatest interference because, despite their presumed acuity disadvantage, they benefited over the near distractors from the end-item advantage that has sometimes been observed in the verbal report of horizontal letter strings (see, e.g., Merikle, Coltheart, \& Lowe, 1971). This seems unlikely, given the well-established effects of proximity in the response-competition paradigm (see, e.g., B. A. Eriksen \& C. W. Eriksen, 1974), and since Driver and Baylis (1989) failed to find any end-item advantage for horizontal arrays with the current spatial and temporal parameters. Nevertheless, we ran a further experiment to examine the possibility of end-item effects. In this experiment, horizontal strings of five letters were again presented as in Experiment 2, but all were in the same color.

\section{EXPERIMENT 3}

The task was again to categorize a central letter at fixation within a display of five letters, which this time were all in the same color.

\section{Method}

Subjects. The 15 subjects, 7 female and 8 male, were lower division psychology undergraduates at the University of California, San Diego. All had normal or corrected-to-normal acuity by selfreport. They received course credit for their participation in this experiment.

Apparatus and Materials. The apparatus was like that in Experiment 1 , except that the stimuli were all presented on an amber monochrome EGA monitor (Samsung). The letters, response assignments, and visual angles were like those in Experiment 2, but all stimuli were amber on a black background. The subjects responded on the " $Z$ " and " " " keys on the standard extended keyboard.

Design. The design was the same as that of Experiment 2, except that since all letters were in the same color, there was no between-subject factor; there were just the five within-subject conditions.

We expected to replicate prior research which showed that, other factors being equal, near incongruent distractors produce more interference than far distractors do (Driver \& Baylis, 1989; B. A. Eriksen \& C. W. Eriksen, 1974). On the other hand, an end-item advantage should lead to more interference from far than from near distractors, just as in Experiment 2, even though the displays in Experiment 3 were monochrome.
Procedure. The procedure was the same as it was in Experiment 1 . The combination of upper and lower RT criteria, derived as for Experiment 1, excluded 3.6\% of the trials.

\section{Results and Discussion}

The means of subjects' median RTs and their mean error rates for the five conditions are shown in Figure 4. A oneway within-subject ANOVA of the RT data showed a significant effect of condition $[F(4,56)=5.54, p<.001$, $M S E=299.2]$. Neuman-Keuls tests revealed that the near incongruent condition was slower than the baseline $(p<$ $.001)$ and the far incongruent $(p<.01)$ conditions, while the latter conditions did not differ significantly.

A similar analysis of the accuracy data showed a significant effect of condition $[F(4,56)=10.1, p<.001$, $M S E=6.44]$. Neuman-Keuls tests found that the near incongruent condition had a higher error rate than did both the baseline $(p<.01)$ and the far incongruent conditions $(p<.05)$, while the latter two conditions did not differ.

These data confirm that in monochrome arrays, incongruent distractors close to the target produce more response competition than do incongruent distractors farther away from the target. This accords with the established
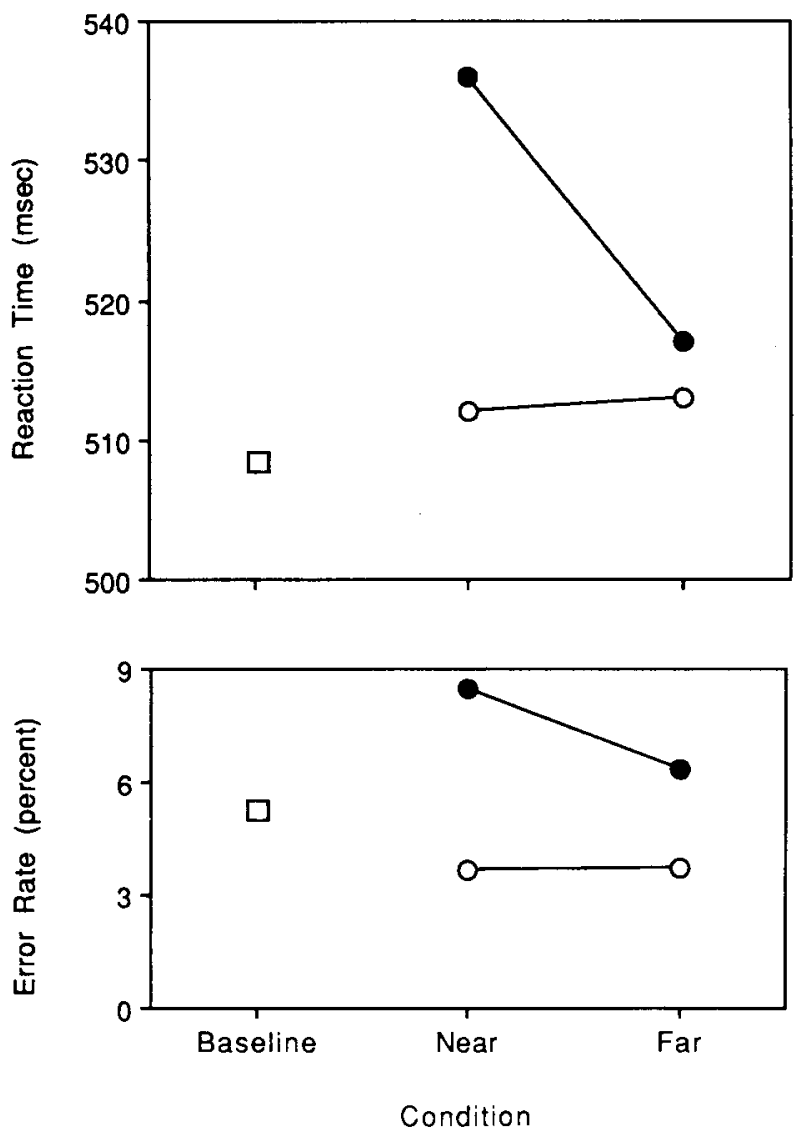

Figure 4. Mean reaction time (above) and error rate (below) for Experiment 3. Congruent conditions are shown in open circles, incongruent in filled circles, baseline as an open square. 
effects of proximity on response competition (see, e.g., B. A. Eriksen \& C. W. Eriksen, 1974), and it shows that conventional proximity effects can be obtained with the present materials, design, and spatial parameters, provided that the target and far distractors are not distinguished from the near distractors by color. Although this distance effect is usually explained in terms of the spotlight or zoom-lens metaphors for attention, according to the current account it would be considered the result of targetdistractor grouping or similarity in proximity.

The distance effect was found in displays that were identical in spatial layout to those used in Experiment 2, in which far distractors in the target's color interfered more than did near distractors in a different color. A pooled analysis of Experiments 2 and 3 confirms the reversal in the pattern of near and far interference. A two-way mixed ANOVA of the RT data, with experiment as the betweensubject factor and the amount of near and far interference (i.e., incongruent near minus baseline, and incongruent far minus baseline) as the within-subject factor, revealed no main effect of experiment $[F(1,33)=.005]$ or of condition $[F(1,33)=0.8]$ but did reveal a powerful interaction $[F(1,33)=16.6, p<.0001]$. A similar analysis of the pooled error data also revealed no main effect of experiment $[F(1,33)=2.3]$ or of condition $[F(1,33)=0.0]$ but did reveal an interaction $[F(1,33)=11.1, p<.002]$. These analyses show that the change in color format from Experiment 2 to Experiment 3 dramatically affected the results for the five within-subject conditions. Indeed, this manipulation had two effects (compare Figures 3 and 4). The use of monochrome displays in Experiment 3 increased the response competition from near distractors, and it decreased the response competition from the far distractors relative to the results of Experiment 2 . The reduced effect of the far distractors in Experiment 3 might at first appear to be paradoxical, since their relation to the target was the same in terms of shared color and proximity as it was in Experiment 2. According to the grouping account, this aspect of the results implies that grouping of the target and distractors is a competitive process. Thus, the more strongly the near distractors are grouped with the target, the less the far distractors can be thus grouped, and vice versa. The notion of competitivity in grouping, which was first noted by Wertheimer $(1923$, p. 313), makes obvious functional sense. Without such competition, grouping would tend to "spread" across a visual scene and would thus fail to segregate the scene.

The reversal in the pattern of near and far response competition for Experiments 2 and 3 can also be accommodated by the similarity account. In Experiment 2, color distinguished the target from the near distractors. Accordingly, color would be used in the target selection process, although one consequence was that the far distractors were relatively hard to ignore since they shared the target color. In Experiment 3, color provided no information to distinguish the target from distractors, and hence the target would be selected by position alone, so that only proximity would determine the impact of distractors.
The pooled analysis of the two experiments emphasizes the contrast between the reverse distance effect found in Experiment 2 and the conventional distance effect of Experiment 3. Given this pattern, the reverse distance effect in Experiment 2 cannot have been due to any enditem advantage. If it had been, a reverse distance effect should also have been found in Experiment 3. However, the far distractors may have produced the most interference in Experiment 2 as the result of some interaction between their being end items and having a color different from that of the near distractors, rather than because they had the same color as that of the target. Experiment 3, which controlled only for the end-item factor, cannot be used to rule out an interaction between this factor and the presentation of near and far distractors in different colors. Experiment 4 was designed to test this possibility.

\section{EXPERIMENT 4}

Experiment 4 required categorization of the central letter in a horizontal array of five letters as before, but this time three colors were used in each display: one for the far distractors, one for the near distractors, and one for the target. If the far distractors in Experiment 2 produced the most interference because they were end items with a color different from that of their nearest neighbors, Experiment 2's pattern of results (i.e., a reverse distance effect) should be found in the new tricolor displays. However, if the pattern of results in Experiment 2 depended on the far distractors' sharing the target's color, it should not be found with the new displays.

Experiment 4 also provided informative data regarding another account of the contrast between Experiments 2 and 3. This invokes possible differences in the spectral sensitivity of the retina at different eccentricities. In Experiment 2, the far distractors were presented in green or purple (and no difference was found between the effects of these colors), whereas in Experiment 3, they were presented in amber. One might therefore speculate that the amber far distractors produced less response competition in Experiment 3 than the purple or green distractors produced in Experiment 2, simply because the retina is less sensitive to the amber region of the color spectrum at the eccentricity of the far distractors. The design of Experiment 4 allowed us to assess the impact of far distractors presented in colors from different regions of the spectrum. In addition to the tricolor displays, monochrome displays like those from Experiment 3 were employed, but they were presented in colors from widely separate parts of the spectrum. These colors included a purple and cyan very similar to the colors used in Experiment 2, and a yellow very similar to the amber used in Experiment 3, thereby permitting a close comparison with those experiments.

\section{Method}

Subjects. The 40 subjects, 23 female and 17 male, were lower division psychology undergraduates at the University of California, San Diego. All had normal or corrected-to-normal acuity and normal cclor vision by self-report. The subjects received course credit for their participation in this experiment. 
Apparatus and Materials. The experiment was conducted on either a Jameco 286 or a Compaq 386/20 microcomputer. The displays were presented on a color VGA monitor (Samsung). The letters, response assignments, añd visual angles were all as in Experiments 2 and 3 . The subjects responded on the " $Z$ " and "/", keys on the standard extended keyboard.

Design. A mixed design was used, with one between-subject factor and two within-subject factors. The between-subject factor, color group, was the arrangement of colors in the tricolor displays. The three possible colors were yellow (Y), cyan (C), and purple (P). The four groups of 10 randomly assigned subjects differed in the sequences of these colors in the horizontal arrays of five letters. The four sequences were as follows: Y CPCY, CY PYC (both these groups also had monochrome displays in purple, so that the target letters appeared in the same color for them throughout the experiment), PCYCP, and CPY PC (the last two groups had monochrome displays in yellow). Different tricolor sequences were used for each group to examine whether any differences between the effects of near and far distractors could be attributed to differences in the salience of the colors chosen for the near and far positions. The first within-subject factor was display color-that is, whether displays were tricolored or monochrome, as described. The second within-subject factor was the interference condition, with five levels corresponding to the five within-subject conditions of Experiments 2 and 3.

Procedure. Events were timed as in Experiment 3. The displaycolor factor was blocked, tricolor or monochrome displays being presented in alternating blocks. The first two practice blocks had 75 trials each. The six experimental blocks that followed had 150 trials each. Half the subjects in each group began with tricolor displays; the other half began with monochrome displays.

Treatment of results. The first 150 trials were discarded as practice, as were the first 2 trials of each block. Thus, 888 trials were available for each subject. All these contributed to the accuracy analyses. Upper and lower RT criteria were applied as before, excluding $6.2 \%$ of the trials.

\section{Results}

The means of subjects' median RTs for the 10 withinsubject conditions are shown in Figure 5, together with their associated mean error rate. The RT data were analyzed with a three-way mixed ANOVA. The betweensubject color-group factor was nonsignificant $[F(3,36)=$ $.5]$, as were the interactions of subject group with display type $[F(3,36)=.87]$ and with interference condition $[F(12,144)=1.3]$. The three-way interaction $[F(12,144)=1.0]$ was also nonsignificant. The data were therefore pooled across the four subject groups. There was no main effect of whether the displays were monochrome or tricolored $[F(1,36)=.8]$. There was a highly significant effect of interference condition $[F(4,144)=$ $40.1, p<.0001]$. Finally, the interaction of display color and interference condition was significant $[F(4,144)=$ $4.5, p<.002]$.

Subsequent Neuman-Keuls comparisons performed on the pooled data $(M S E=361.0)$ showed that, in monochrome displays, the incongruent near condition was slower than both the baseline and the incongruent far conditions ( $p<.001$ in both cases), but that the latter two conditions did not differ. Thus, the conventional Eriksen distance effect (more response competition from near than from far distractors) was observed in both the yellow and the purple monochrome displays, and the reversal of the
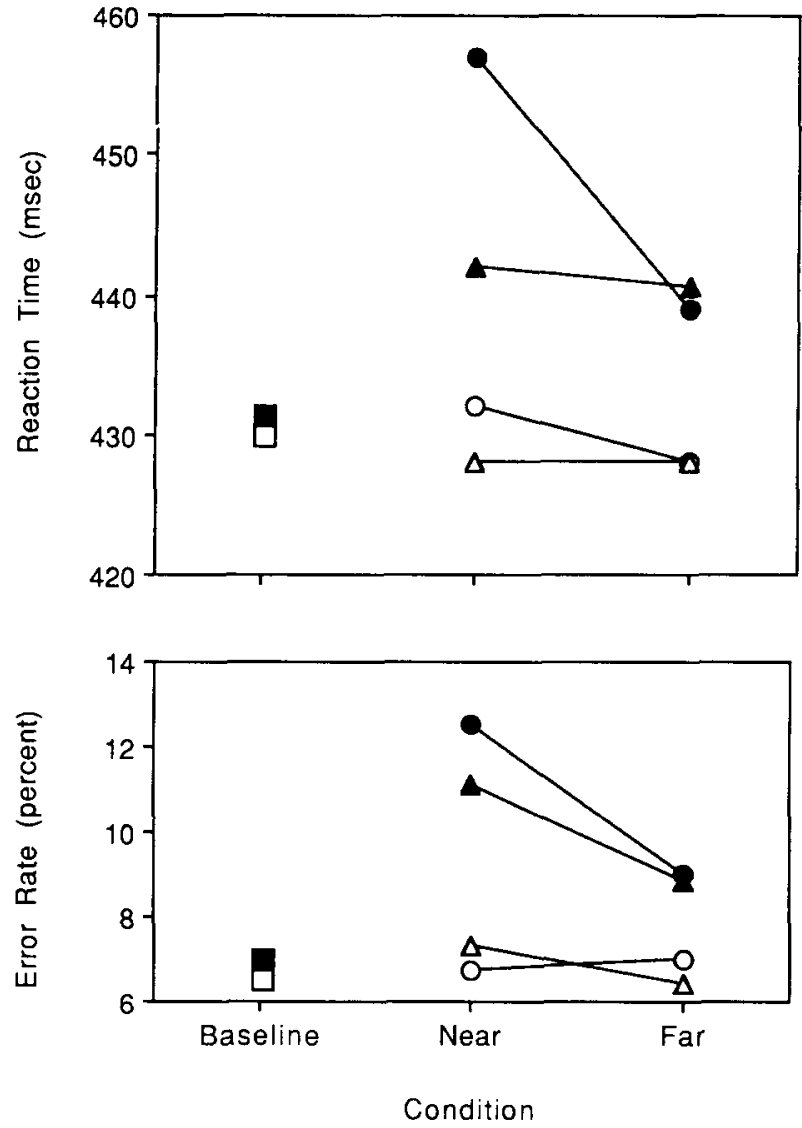

Figure 5. Mean reaction time (above) and error rate (below) for Experiment 4. Congruent conditions are shown in open symbols, incongruent in filled symbols. Results for tricolored displays are shown as triangles; results for monochrome displays are shown as circles. The tricolor baseline is shown as a filled square, the monochrome baseline as an open square.

distance effect in Experiment 2 as opposed to Experiment 3 cannot be attributed to the particular color used for the far distractors in Experiment 2 (purple or green) as opposed to Experiment 3 (amber).

A similar pattern of results was found for the tricolor displays. The incongruent near condition was significantly slower than the baseline $(p<.05)$, but no other comparison reached significance. Inspection of Figure 5 suggests that the incongruent near distractors produced less of an effect than they did in the monochrome displays. To test for such a difference, the response competition due to near distractors was calculated for each subject (i.e., median RT to the near incongruent displays minus median RT to the baseline displays). Each subject yielded two such measures-one for the monochrome and one for the tricolor displays. These data were analyzed in a twoway mixed ANOVA, the between-subject factor being color group as before, and the within-subject factor monochrome versus tricolor displays. There was no effect of color group $[F(3,36)=0.7]$; there was a main effect of monochrome as opposed to tricolor displays $[F(1,36)=$ 
$8.1, p<.01]$; and there was no interaction $[F(3,36)=$ 1.8]. This supports the contention that the interfering effect of the near distractors is reduced in the tricolored displays in comparison with their effect in the monochrome displays, even though more interference from near than from far distractors is found in both cases.

A three-way mixed ANOVA on the error data from all conditions supports the conclusions from the RT data. The between-subject factor was nonsignificant $[F(3,36)=$ $.50]$, as were its interactions with display color $[F(3,36)$ $=.54]$ and with interference condition $[F(12,144)=.75]$. The three-way interaction $[F(12,144)=.75]$ was also nonsignificant. The data were therefore pooled across the four subject groups, and the mean error rates for the 10 withinsubject conditions are shown in Figure 5. There was no effect of whether the displays were tricolor or monochrome $[F(1,36)=0]$. There was a highly significant effect of interference condition $[F(4,144)=29.8, p<.001]$. Finally, the interaction of display color and interference condition was nonsignificant $[F(4,144)=1.3]$.

Subsequent Neuman-Keuls comparisons $(M S E=21.2)$ showed that in monochrome displays there were more errors for the incongruent near condition than for the baseline $(p<.001)$ or the incongruent far condition $(p<.001)$ and that the latter conditions did not differ. In the tricolor displays, there was a higher error rate in the incongruent near condition than in the baseline $(p<.001)$ and the incongruent far $(p<.05)$ conditions, but the latter conditions did not differ.

The size of the response-competition effect on accuracy due to the near distractors (i.e., the near incongruent condition minus the baseline) in the tricolor and in the monochrome displays was compared in a two-way mixed ANOVA (color group $\times$ display type) as before. The difference in this response competition for tricolor as opposed to monochrome displays was close to significance $[F(1,36)$ $=3.8, p<.06]$. There was no effect of group $[F(3,36)=$ $1.1]$, nor was there an interaction $[F(3,36)=1.1]$.

\section{Discussion}

The results from the monochrome displays demonstrate that the conventional effect of proximity on response competition (i.e., more interference from near distractors) is robust across changes in display color from amber to purple or yellow. Hence, the finding in Experiment 2 that purple or green far distractors caused more interference than did near distractors cannot be attributed to variations in spectral sensitivity across the retina.

The far distractors in the tricolor displays appeared as end items with a color different from that of their nearest neighbors. However, unlike the far distractors of Experiment 2 , they did not produce more response competition than did the near distractors. Thus, the reverse distance effect in Experiment 2 should be attributed to the fact that the far distractors shared the target's color, rather than to the fact that they had a color different from that of adjacent items.

The near distractors produced less response competition in the tricolor displays than in the monochrome dis- plays. This effect is reminiscent of the results of Experiment 1 , in which distractors at a constant separation from the target produced more interference if they shared the target color. A closer analogy for the present tricolor as opposed to monochrome effect can be found in the data of Harms and Bundesen (1983), who also observed more interference in monochrome displays than in displays in which target and distractors differed in color. According to the grouping account, in monochrome displays, the target and distractors are grouped by proximity plus common color, rather than by proximity alone. According to the similarity account, subjects can select the target by color in addition to position in the multicolor case, but they can only use position to distinguish targets and distractors in monochrome displays, resulting in less efficient selection.

\section{SUMMARY OF EXPERIMENTS 1-4}

In Experiment 1, the displays contained four distractors at a constant separation from the target, two of which matched the target in color, and two of which had a different color. More response competition was found from incongruent distractors when they shared the target's color. In Experiment 2, it was found that, with appropriate distance and color parameters, the effects of color could be sufficiently powerful to override the effects of proximity, so that relatively distant distractors in the target's color produced more response competition than did distractors that were closer but had a different color. Experiments 3 and 4 showed, respectively, that this result was not attributable to the far distractors' position at either end of a horizontal letter string or to their appearing as end items with a color different from that of their nearest neighbors. The results of Experiment 2 are analogous to Driver and Baylis's (1989) finding that the factor of motion overrode proximity in determining the extent of response competition. They imply that visual attention is not directed on the basis of position alone, even in static displays when target location is known in advance.

As discussed earlier, Harms and Bundesen (1983) found more interference in monochrome displays than in multicolor displays, a result that we also obtained in the comparison of tricolor and monochrome displays in Experiment 4 . This finding is consistent with the grouping or similarity accounts we have outlined. However, with the parameters that Harms and Bundesen explored, color had a relatively small effect on response competition, which was only apparent in error rates. We will now consider possible reasons for the apparent discrepancy between the size of the color effects Harms and Bundesen observed and those we found in Experiments 1 and 2. These possibilities include the particular colors employed, the involvement of competitive processes in grouping, and whether or not the color of the target is constant. Further experiments will be required to distinguish the roles of these factors.

The visual search paradigm provides evidence that the relative effects of color and proximity on the distribution 
of visual attention depend heavily on the particular color parameters involved. Initial results suggested that, contrary to our conclusions from Experiment 2, attention could not be directed to dispersed items with a common color to the relative exclusion of interleaved items in a different color. Treisman and Gelade (1980) originally found that visual search for a conjunction of color and form (e.g., a red $\mathrm{X}$ in intermingled green $\mathrm{Xs}$ and red Os) was serial, suggesting that visual attention could not readily be applied to a spatially dispersed common color group (the red items). If Treisman and Gelade's subjects had been able to restrict themselves to this color group, their conjunction task should have turned into a parallel feature search for the red $\mathrm{X}$ among red Os. Follow-up work by Treisman (1982) appeared to confirm the conclusion. She found that the conjunction task became parallel only when commonly colored items were arranged contiguously, suggesting that attention could be applied to a unitary region of space, but not to a spatially dispersed common-color group. However, Wolfe, Cave, and Franzel (1989) have since found cases of parallel color-form conjunction search, indicating that attention can be assigned to spatially dispersed items with a common color under some circumstances. Whether or not this is possible now seems to depend on the discriminability of the colors involved (Treisman, 1988). Thus, the recent visual search data corroborate our conclusion in Experiment 2 that visual attention can in some circumstances be applied to commonly colored items to the relative exclusion of interleaved items in a different color, but we must emphasize that this phenomenon is likely to depend heavily on the particular colors used.

Harms and Bundesen (1983) used a between-display manipulation of target-distractor similarity in color, whereas in Experiments 1 and 2 we used a within-display manipulation. That is, we presented distractors that shared the target color at the same time as we presented distractors that did not. Recall that when we compared Experiments 2 and 3, we found that an increase in response competition from the near distractors coincided with a decrease in interference from the far distractors, which suggested that grouping (or target-distractor similarity) is a competitive process. Presumably this competition takes place between the items within a particular display. In Experiments 1 and 2, we examined the effects of distractors that shared the target color as they competed with distractors from the same display that had a different color. However, in the Harms and Bundesen (1983) study (and in the present Experiment 4, which also yielded a relatively small effect of color in the tricolor vs. monochrome displays), distractors sharing the target's color and those having a distinct color were compared between displays. Thus, different-color distractors never had to compete directly against same-color distractors. Such competition might be expected to amplify any difference in their effects. We return to the issues of competition for grouping and between- versus within-display manipulations with our final experiment.
A final difference between the present experiments and those of Harms and Bundesen (1983) is that the target color was constant for any subject in our studies, whereas it was unpredictable in the Harms and Bundesen experiments. The work of Humphreys (1981, Experiment 2) and Tipper (1985) suggests that selection is more efficient when the color of the target is held constant. One possibility is that subjects may use color as a selection cue only when the color of the target is predictable, as suggested by Humphreys (1981). According to this view, the effects of color on response competition in Experiments 1 and 2 would reflect the strategic use of color in the selection process rather than the inevitable consequences of bottomup grouping processes. We intend to examine this strategic account by repeating Experiments 1 and 2 with unpredictable target colors. However, another perspective makes predictions similar to those based on the strategic account-in particular the prediction that the effects of color on response competition should be attenuated when target color is unpredictable. This learning view stresses that during the course of the experiment, responses are learned with respect to targets that have a certain set of attributes, such as positions, shapes, motions, and colors. Incongruent distractors elicit competing responses to the extent that they match previously experienced targets with associated responses (B. A. Eriksen \& C. W. Eriksen, 1974; C. W. Eriksen \& St. James, 1986; Yeh \& Eriksen, 1984). Thus, for a subject who has only experienced green targets, incongruent green distractors should produce more interference than incongruent red distractors would produce (as we found in Experiment 1), simply because they are more similar to the targets from previous displays. We plan to examine this possibility by having a target color that is known in advance (and that therefore should be used strategically) but that can be changed from trial to trial to manipulate prior learning experiences.

Whatever the resolution of these issues, Experiments 1-4 demonstrate that proximity is not the only targetdistractor relationship that affects the amount of response competition from incongruent distractors, because color can have analogous effects. In our next experiments, we examined whether any other static factors have effects similar to those of color and proximity. Inspection of the displays from Experiment 1 (see, e.g., Figure 1a) suggests one possibility. The diagonal with common color appears to form a good continuation group. In Experiments 5 and 6 , we examined whether this factor alone could influence the response competition produced by incongruent distractors at a constant distance from the target.

\section{EXPERIMENT 5}

The task was again to categorize a central letter appearing at fixation. In this experiment, the target was surrounded by four distractor letters-one above, one below, one to the left, and one to the right-to yield a crossshaped format. These five letters were embedded in a rect- 
angular array of horizontal strokes that grouped items into rows rather than columns by the principle of good continuation (see Figure 1c). If this grouping factor affects response competition, there should be more interference from the horizontal as opposed to the vertical distractors, because only the former were grouped into the target's row by good continuation.

\section{Method}

Subjects. The subjects were 25 lower division undergraduates at the University of California, San Diego, of whom 14 were female, 11 male. All had normal or corrected-to-normal acuity by self-report, and all received course credit for participating in this experiment.

Materials. The letters and response assignments were the same as they were in the previous experiments. This time, however, each display contained 5 letters presented in a cross format and embedded in an array of horizontal strokes; there were 15 items in all, arranged in three rows and five columns (see Figure 1c). All distractor letters were equidistant from the central target letter. The visual angles were the same as those in Experiments 1, 3, and 4 for the letters. The horizontal strokes were spaced like the letters, and the entire display subtended $3.5^{\circ} \times 2.0^{\circ}$. The equipment was the same as that used in Experiments 1, 3, and 4. The letter distractors in a row were always repetitions of a single item, as were the letter distractors in a column.

Design. In Experiment 5, items were grouped into rows by using the principle of good continuation. We investigated whether distractors in the row that the target was in would cause greater interference than would distractors in a different row. The design was within subjects, using a factor analogous to that in the preceding experiments. This was the identity and location of target and distractors, leading to five conditions:

Baseline. All letters in the display except the target were neutral (i.e., $\mathbf{H}$ or $\mathrm{T}$ ).

Congruent, same row. Letters in the row that the target was in were congruent with the target item; letters above and below the target were neutral.

Congruent, different row. Letters in the row that the target was in were neutral; those above and below were congruent with the target.

Incongruent, same row. Letters in the row that the target was in were incongruent with the target; those above and below were neutral.

Incongruent, different row. Letters in the row that the target was in were neutral; those above and below were incongruent with the target.

Procedure. Except as noted, the procedure followed Experiment 1 . The subjects were shown a scale diagram of the displays, and it was explained that the displays consisted of "three rows of five items," with the target "always in the center of the middle row." The subjects were encouraged to ignore everything except the central target, and to respond as quickly as possible while maintaining accuracy.

Treatment of results. This was the same as it was in Experiments 1,3 , and 4 , with $2.7 \%$ of the trials excluded from the RT analysis by the imposition of lower and upper cutoffs.

\section{Results}

The mean of subjects' median RTs and their mean error rates are shown in Figure 6. A one-way within-subject ANOVA of the RT data showed a significant effect of condition $[F(4,96)=17.4, p<.001, M S E=79.1]$. Neuman-Keuls comparisons revealed that the incongruent same-row condition was slower than the baseline $(p<$ $.001)$ and incongruent different-row conditions $(p<.01)$. A similar analysis was carried out on the accuracy data,
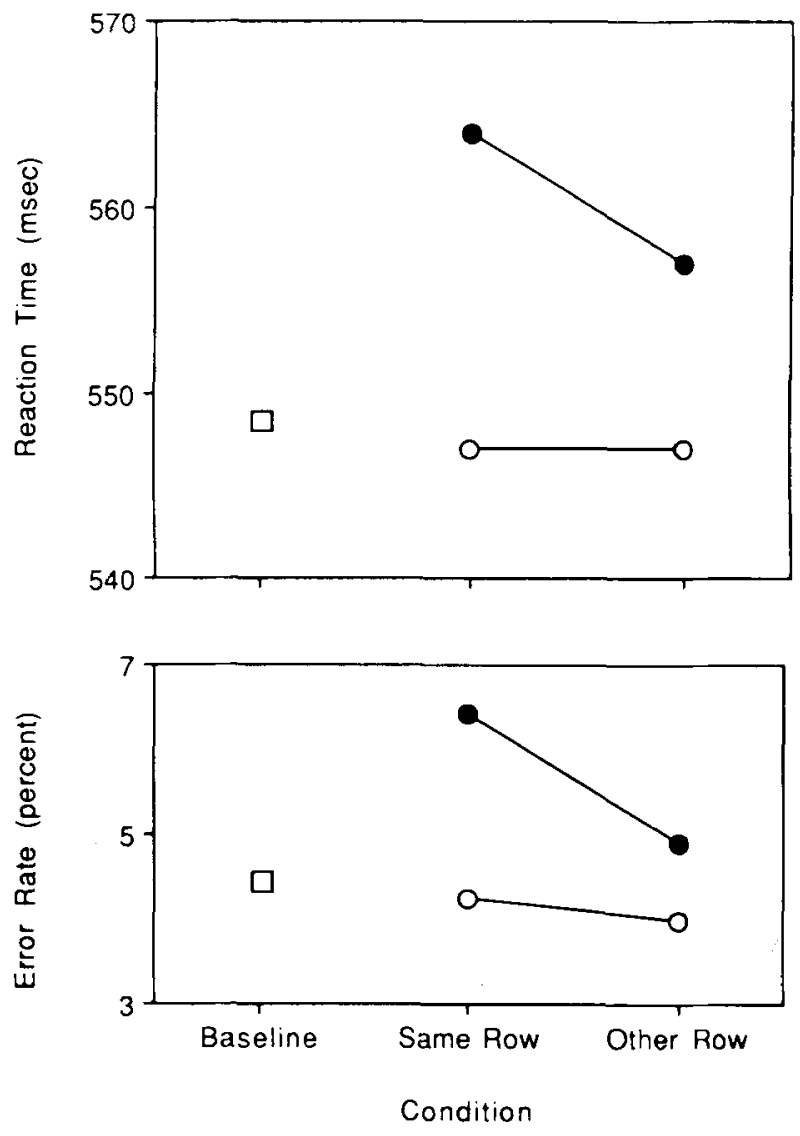

Figure 6. Mean reaction time (above) and error rate (below) for Experiment 5. Congruent conditions are shown in open circles, incongruent in filled circles, baseline as an open square.

showing that there was again a significant effect of condition $[F(4,96)=6.8, p<.01, M S E=3.54]$. NeumanKeuls comparisons revealed that there were more errors in the incongruent same-row condition than in the baseline $(p<.001)$ and incongruent different-row conditions $(p<.01)$, but that the latter conditions did not differ.

\section{Discussion}

In a display grouped into rows by good continuation, more response competition was observed from the distractors that were in the target's row. The distractors in different rows produced less interference, even though they were equally close to the target. This suggests that good continuation affects response competition. However, it is possible that the greater interference from the horizontally aligned distractors was not caused by good continuation. Extended reading experience might be responsible for the tendency to organize letters into rows rather than columns irrespective of good continuation, since words are usually encountered as horizontal letter strings. In Experiment 6, we used good continuation to organize the display into columns. If the results of Experiment 5 reflect a tendency to organize letters horizontally irrespective of good continuation, the same pattern of results should be 
observed with the columnar organization. However, if the results do reflect good continuation, the pattern should reverse so that more response competition is observed from the verticaldistractors in the column with the target.

\section{EXPERIMENT 6}

This experiment was similar to Experiment 5, except that the five letters were embedded in a rectangular array of vertical strokes that grouped items into columns rather than rows by the principle of good continuation (see Figure 1d).

\section{Method}

Subjects. The subjects were 30 lower division undergraduates at the University of California, San Diego, of whom 18 were female, 12 male. All had normal or corrected-to-normal acuity by self-report, and all received course credit for participating in this experiment.

Materials. All aspects of the materials were the same as they had been in Experiment 5, except that each display contained five letters, presented in a cross format as before but now embedded in an array of vertical strokes organized into three columns (see Figure 1d).

Design. Items were grouped into columns according to the principle of good continuation. By way of analogy with Experiment 5, we investigated whether distractors in the target's column would have greater interfering effects than would those in a different column. The design was within subjects, using a factor analogous to that in Experiment 5, leading to five conditions:

Baseline. All letters in the display except the target were neutral (i.e., $\mathrm{H}$ or $\mathrm{T}$ ).

Congruent, same column. Letters within the target's column were congruent with the target; letters to the left and right (i.e., in different columns) were neutral.

Congruent, different column. Letters in the target's column were neutral; those in different columns were congruent with the target.

Incongruent, same column. Letters in the target's column were incongruent with the target; those in different columns were neutral.

Incongruent, different column. Letters in the target's column were neutral; those in different columns were incongruent with the target.

Procedure and treatment of results. These were all as in Experiments 1 and $3-5$, with $3.5 \%$ of the trials excluded from the RT analysis by the lower and upper cutoffs.

\section{Results}

The means of subjects' median RTs and their mean error rates are shown in Figure 7. A one-way within-subject ANOVA of the RT data showed a significant effect of condition $[F(4,116)=8.8, p<.001, M S E=97.3]$. Neuman-Keuls tests revealed that the incongruent samecolumn condition was slower than the baseline $(p<.001)$ and incongruent different-column $(p<.001)$ conditions. A similar analysis of the error data yielded an effect of condition $[F(4,116)=3.1, p<.02, M S E=4.45]$. The only significant Neuman-Keuls comparison in these data was that more errors were found in the incongruent samecolumn condition than in the baseline condition $(p<.05)$.

\section{Discussion}

When the display was grouped by good continuation into columns, rather than into rows as in Experiment 5, more response competition was observed from the verti-
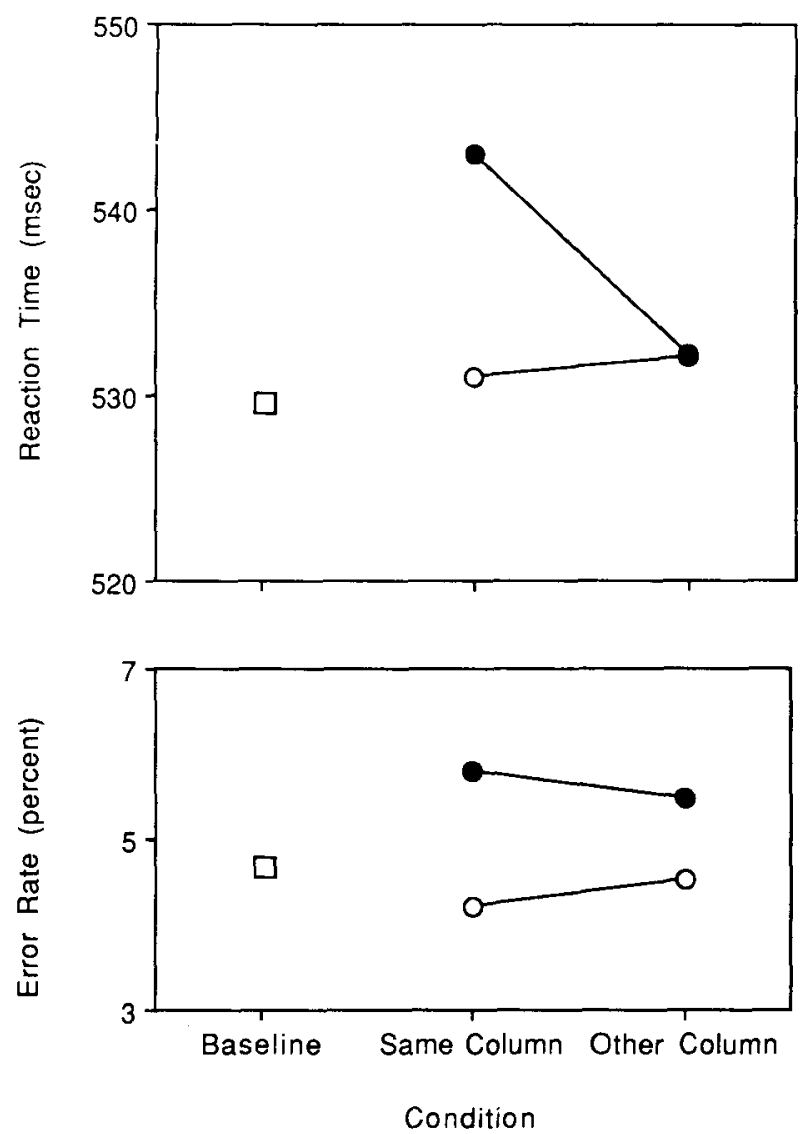

Figure 7. Mean reaction time (above) and error rate (below) for Experiment 6. Congruent conditions are shown in open circles, incongruent in filled circles, baseline as an open square. Note that the labeling is in terms of columns, whereas Figure 6 was labeled in terms of rows.

cal distractors. The horizontal distractors, which had exerted the greatest influence in Experiment 5 but were now in different columns from the target's, produced less interference even though they were equally close to the target. This supports a role for good continuation in response competition, demonstrating that color is not the only static factor besides proximity that affects the amount of interference from incongruent distractors.

As we have stressed, our observations that color affects response competition can be accommodated either by the view that visual attention is assigned to perceptual groups, or in terms of target-distractor similarity. According to the latter view, the more similar a distractor is to the target on the attributes used for location (e.g., a particular location and/or color), the harder the distractor is to reject, regardless of how the display is organized perceptually. The grouping and similarity views are difficult to distinguish, if they are rivals at all, since most Gestalt grouping principles operate according to similarity. Indeed, in his description of factors that lead to perceptual grouping, Wertheimer (1923, pp. 308-309) considered common color a subtype of the similarity factor. Neverthe- 
less, the grouping hypothesis provides a more natural explanation for the results of Experiments 5 and 6 than does the similarity account. The horizontal distractors in Experiment 5 are only "more similar" to the target than the vertical distractors because they appear in the same row and the display is organized into rows. Likewise, the reverse similarity can only apply in Experiment 6, because these displays are organized into columns. In other words, an account of these data in terms of target-distractor similarity would simply be a restatement of the grouping position.

A reviewer suggested that the results of Experiments 5 and 6 might reflect different scanning patterns-presumably, given the patterns of interference, horizontal scanning in Experiment 5 and vertical in Experiment 6. Since the target always appeared at fixation, it is not clear why a scan would be required at all. Because the displays were only $180 \mathrm{msec}$ in duration, any scanning that might have taken place would have to have involved covert attention rather than overt eye movements. Moreover, the only reason for vertical scanning to have occurred in Experiment 6 and for horizontal scanning to have occurred in Experiment 5 would have been the perceptual organization of the displays into columns or rows. Thus, the covert scanning account does not contradict the grouping account, but rather provides a possible explanation of why within-group interference is greater once the display has been parsed.

Experiment 6, with its columnar organization, demonstrates that good continuation can overcome any tendency that may exist, as a result of reading experience perhaps, for organizing letters into rows rather than columns. In the next experiment, we specifically tested whether such a horizontal preference would exist in the absence of good continuation factors.

\section{EXPERIMENT 7}

The task was to categorize a central letter at fixation surrounded by four distractors in a cross-shaped format as before. However, these letters were not embedded in an array of horizontal or vertical strokes as in Experiments 5 and 6 (see Figure le). We could therefore examine whether there would be more interference from horizontal than from vertical distractors when good continuation factors were neutral.

\section{Method}

Subjects. The subjects were 25 lower division undergraduates of the University of California, San Diego, 16 of whom were female, 9 male.

Materials. All aspects of the materials were the same as in Experiments 5 and 6, except that each display contained only the five letters-that is, there were no distracting dashes (see Figure le).

Design. In this experiment, we investigated whether in the absence of powerful good continuation factors there is a residual tendency to show more response competition from horizontally aligned rather than vertically aligned distractor letters. The design was within subjects, using a factor analogous to that in Experiments 5 and 6, leading to five conditions:
Baseline. All items in the display except the target were neutral (i.e., $\mathrm{H}$ or $\mathrm{T}$ ).

Congruent horizontal. Distractors in the row were congruent with the target; distractors in the column were neutral.

Congruent vertical. Distractors in the row were neutral; those in the column were congruent.

Incongruent horizontal. Distractors in the row were incongruent with the target; those in the column were neutral.

Incongruent vertical. Distractors in the column were incongruent; those in the row were neutral.

Procedure and treatment of results. These were as in Experiments 1 and 3-6, with 3.4\% of all the trials excluded from the RT analysis by the imposition of upper and lower cutoffs.

\section{Results}

The means of subjects' median RTs and their mean error rates are shown in Figure 8. A one-way within-subject ANOVA of the RT data showed a significant effect of condition $[F(4,96)=12.5, p<.001, M S E=112.6]$. Neuman-Keuls comparisons revealed that the incongruent horizontal condition was slower than the baseline $(p<.001)$ and incongruent vertical $(p<.02)$ conditions, and that the incongruent vertical was also slower than the baseline $(p<.01)$. A similar analysis of the ac-
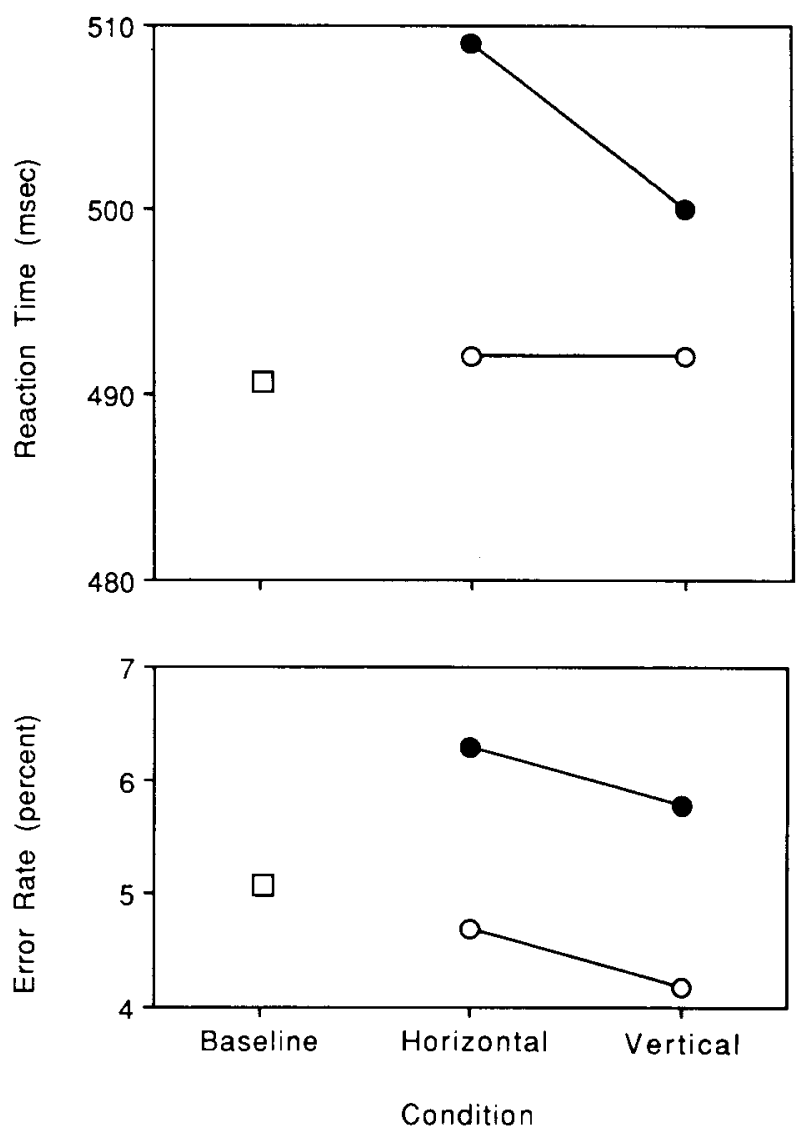

Figure 8. Mean reaction time (above) and error rate (below) for Experiment 7. Congruent conditions are shown in open circles, incongruent in filled circles, baseline as an open square. 
curacy data revealed an effect of condition $[F(4,96)=$ $7.3, p<.001, M S E=2.45$ ], with more errors in the incongruent horizontal condition than in the baseline condition $(p<.01)$.

\section{Discussion}

Incongruent horizontal distractors produced more interference than did incongruent vertical distractors, even though the latter were just as close to the target and good continuation factors were equivalent horizontally and vertically. This suggests that observers have a tendency to group or covertly scan letters horizontally rather than vertically. It is tempting to speculate that this effect may be related to extensive reading experience with text presented in horizontal lines.

In our final experiment, we again examined whether horizontally aligned distractors produce more response competition than vertically aligned distractors do, but we used a between-display manipulation rather than a withindisplay manipulation. We noted earlier that the color data from Experiments 2 and 3 suggest that the grouping of different distractors with the target is a competitive process within each display. If so, within-display manipulations of target-distractor grouping should produce more powerful effects than should analogous between-display manipulations. Any difference between the interfering effects of distractors that share some attribute with the target and the interfering effects of distractors that do not should be more pronounced if the two types of distractor are set against each other in the same display and allowed to compete for grouping with the target. Another way to express this is to say that distractors that do not share the attribute in question may nevertheless group strongly with the target if they are the only distractors, because they will be linked to the target by simultaneous onset on the same display screen, so that there can be no better candidates to group with the target.

In Experiment 8, we compared the distracting effects of incongruent letters presented in the target's row with the distracting effects of incongruent letters presented in the target's column, but this time only one of the two possibilities could be present on any trial-three letters were presented in each display, arranged either in a column or in a row. Experiment 7 had resulted in more interference from distractors in a row with the target than from distractors in a column with the target in the same display. On the basis of this result, one might expect to find more response competition from horizontal than from vertical distractors in Experiment 8. However, recall that when a columnar organization was imposed on the display (in Experiment 6), vertically aligned distractors did produce substantial response competition. In Experiment 8 , only one organization was possible for each display. There was nothing to compete with the columnar organization or with the organization into rows, unlike in the displays of Experiment 7, which could in principle be parsed either way. On these grounds, one might expect that vertically aligned distractors could produce as much response competition as could horizontally aligned distractors in Experiment 8.

\section{EXPERIMENT 8}

The task was to categorize a central letter at fixation either presented alone or surrounded by two distractors, both in a row or both in a column with the target. As before, we examined whether there would be more response competition from horizontal than from vertical incongruent distractors. In addition, we examined whether any nonspecific interference (e.g., Bouma, 1970) from the presence as opposed to the absence of neutral distractors would be greater when they were in a row with the target.

\section{Method}

Subjects. The subjects were 25 lower division undergraduates at the University of California, San Diego, 14 of whom were female, 11 male. All had normal or corrected-to-normal acuity by self-report, and all received course credit for participating in this experiment.

Materials. All aspects of the materials were as in Experiment 7 , except that each display that included distractors contained only three letters, arranged into a column or into a row (see Figure 1f), and no-distractor displays were also presented as a baseline for measuring any effects of the presence as opposed to the absence of distractors per se.

Design. In this experiment, we again investigated whether there would be a tendency to organize letters into rows rather than into columns, in this case when only one of the two possibilities was present on any trial. The design allowed us to investigate both response competition from incongruent distractors as before and the lower level interference (see, e.g., Bouma, 1970) produced simply by the presence of neutral distractors measured relative to a baseline in which the target was presented alone. The design was within subjects, with seven conditions:

No distractor. The target was presented alone.

Congruent horizontal. Congruent letters were presented in a row with the target.

Congruent vertical. Congruent letters were presented in a column with the target.

Horizontal baseline. Neutral letters were presented in a row with the target.

Vertical baseline. Neutral letters were presented in a column with the target.

Incongruent horizontal. Incongruent letters were presented in a row with the target.

Incongruent vertical. Incongruent letters were presented in a column with the target.

Comparison of the incongruent conditions with their corresponding (i.e., horizontal or vertical) baseline conditions yields a measure of response competition. Comparison of the baseline conditions with the no-distractor condition gives a measure of any interference caused by the mere presence of neutral distracting letters.

Procedure and treatment of results. These were all as in Experiments 1 and 3-7, with $3.2 \%$ of trials being excluded from the RT analysis by the lower and upper cutoffs.

\section{Results}

The means of subjects' median RTs and their mean error rates are shown in Figure 9. A one-way within-subject ANOVA of the RT data showed a significant effect of condition $[F(6,144)=11.3, p<.001, M S E=156.4]$. 

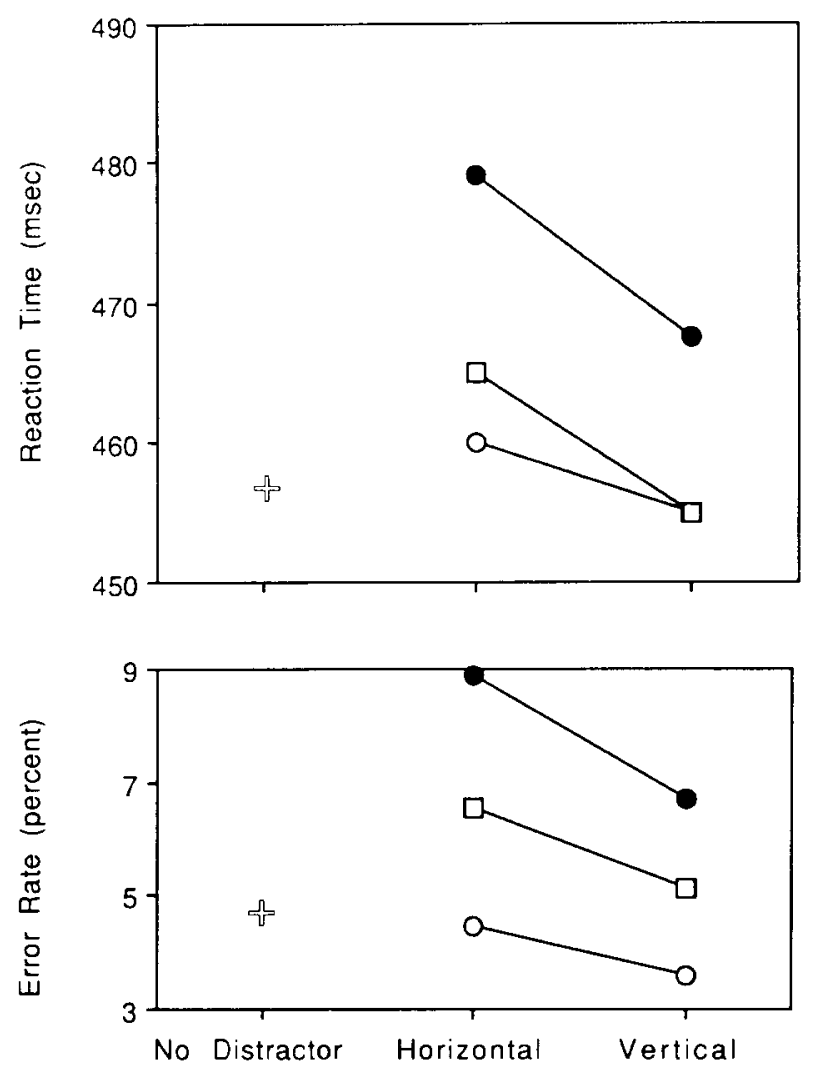

Condition

Figure 9. Mean reaction time (above) and error rate (below) for Experiment 8. Congruent conditions are shown in open circles, incongruent in filled circles, baselines as open squares, and the nodistractor condition is shown as a cross.

Neuman-Keuls tests revealed that the incongruent vertical condition was slower than the vertical baseline $(p<.01)$ and that the incongruent horizontal condition was slower than the horizontal baseline $(p<.01)$.

Inspection of Figure 9 suggests that, in contrast with the results of Experiment 7, vertical distractors produced as much response competition as did horizontal distractors. This was confirmed by further analyses in which the size of horizontal response competition was compared with the size of vertical response competition for each subject. To make this comparison, a two-way ANOVA was performed on four conditions only: incongruent vertical, incongruent horizontal, vertical baseline, and horizontal baseline. This analysis revealed a highly significant effect of incongruent as opposed to baseline $[F(1,24)=$ $22.9, p<.001]$ and of horizontal as opposed to vertical $[F(1,24)=20.65, p<.001]$, but no interaction $[F(1,24)$ $=0.25]$. The same result can be seen if the incongruent conditions are compared with the congruent conditions instead of the baselines in an analogous two-way ANOVA. This revealed a highly significant effect of incongruent as opposed to congruent $[F(1,24)=37.6, p<.001]$ and of horizontal as opposed to vertical $[F(1,24)=11.45$, $p<.01]$, but no interaction $[F(1,24)=2.0]$. Presumably the difference between the patterns of response competition for Experiments 7 and 8 arose because only one organization (column or row) was possible for each display in Experiment 8. Unlike in Experiment 7, the two organizations were not allowed to compete as rivals within a single display.

However, there was a difference in the low-level (i.e., not identity-specific) interference produced by vertical and horizontal distractors, with more interference in the latter case. The only differences between the baselines and the no-distractor condition were that the horizontal baseline was slower than the no-distractor condition $(p<.05)$ and the vertical baseline $(p<.05)$. Thus, nonspecific interference attributable to lateral masking was only seen with horizontal distractors.

Similar analysis of the accuracy data reveals an analogous pattern of results. The ANOVA revealed a main effect of condition $[F(6,144)=16.1, p<.001, M S E=$ 4.84]. Neuman-Keuls tests revealed that the incongruent vertical condition had more errors than did the vertical baseline $(p<.01)$ and that the incongruent horizontal had more errors than did the horizontal baseline $(p<.01)$. In further agreement with the RT data, more low-level interference was found from items that were horizontally rather than vertically aligned with the target. The horizontal baseline had more errors than did the vertical baseline $(p<.05)$ and the no-distractor condition $(p<.05)$, but no other differences were significant.

As with the RT data, vertical distractors produced as much response competition as did horizontal distractors. A two-way ANOVA restricted to the incongruent and baseline conditions again showed a highly significant effect of incongruent as opposed to baseline $[F(1,24)=27.3$, $p<.001]$ and of horizontal as opposed to vertical $[F(1,24)=26.9, p<.001]$, but no interaction $[F(1,24)=$ $1.0]$. As with the RT data, the same result can be seen if the incongruent conditions are compared with the congruent conditions instead of the baselines in a two-way ANOVA. This showed a highly significant effect of incongruent as opposed to congruent $[F(1,24)=34.4, p<.001]$ and of horizontal as opposed to vertical $[F(1,24)=16.0$, $p<.001]$, but no interaction $[F(1,24)=3.4]$.

\section{Discussion}

In contrast with the results of Experiment 7, incongruent distractors arranged in a column with the target produced as much response competition as did distractors arranged in a row with the target. Presumably, this difference arose because only one organization, column or row, was possible for each display in Experiment 8. This supports the notion of competitive target-distractor grouping suggested by Experiments 2 and 3 and our discussion of the Harms and Bundesen (1983) data. It seems that the relative strength of target-distractor grouping within a display may be as important as the absolute level in determining the interference of a particular distractor. 
Thus, the incongruent vertical distractors produced robust response competition in Experiment 8 and so did the columnar displays in Expetiment 6, because no other distractors in the same display were grouped more strongly with the target. However, incongruent distractors with the very same relationship with the target produced less of an effect in Experiments 5 and 7, because, in these displays, the horizontal distractors were more strongly grouped with the target.

Although response competition was equivalent from the vertical and horizontal distractors, more low-level interference (i.e., an impairment caused by the mere presence of distractors rather than by their identity) was produced by horizontal distractors. We can only speculate about the origin of this effect. Again, it would be interesting to examine its relation to reading.

\section{GENERAL DISCUSSION}

Our results demonstrate that the amount of response competition from incongruent distractors in static displays is not solely determined by their distance from the target. Distractors that share the target's color produce more interference than do those that do not (Experiment 1), and the effects of color can be sufficiently strong to override those of proximity under some circumstances (Experiment 2). Distractors grouped with the target by good continuation produce more response competition than do those that are equally close but in a different good-continuation group (Experiments 5 and 6). These findings suggest that visual attention is directed to groups derived from a segmentation of the scene according to Gestalt principles of perceptual organization, rather than directed to unparsed regions of the visual field. They imply that attention is not directed on the basis of position information alone (cf. Tsal \& Lavie, 1988), even when the position of the target is known in advance.

An alternative to the grouping account of the observed effects of color is that the amount of response competition from distractors is simply a function of target-distractor similarity, irrespective of how the display is parsed. However, this hypothesis does not provide a natural account of our data on the effects of good continuation, because the target-distractor similarities required to explain the pattern of response competition appear to follow only from the way in which the displays are organized: items within a good-continuation group would have to be more "similar" than would those in different good-continuation groups. The grouping account is therefore preferable on the grounds of parsimony. The suggestion that visual attention is directed to perceptual groups also makes considerable functional sense. Following many others (e.g., Marr, 1982), we suppose that the Gestalt principles of perceptual organization reflect parsing operations in early vision. These have presumably evolved to link the elements of a visual scene that are likely to belong together in the real world-that is, likely to be components of a single object. Since organisms direct their actions toward individual objects, it is presumably adaptive for selective attention to operate on perceptual units that are likely to capture real-world divisions between different objects.

Components of a single object will often occupy adjacent regions of visual space. Given the view advocated here, this is one reason why proximity is a powerful grouping factor that exerts strong effects on visual attention (see, e.g., B. A. Eriksen \& C. W. Eriksen, 1974; cf. the present Experiments 3 and 4). However, there are cases in which components of a single object will be spatially intermingled with those of another object (e.g., an animal behind partially occluding foliage). We suppose that in these instances, nonspatial grouping principles such as common color operate to segregate relevant and irrelevant objects so that attention may be directed to the former. Rock and Gutman (1981) and Tipper (1985) have demonstrated that visual selection in human observers is highly efficient even when the relevant object is a line drawing superimposed on an irrelevant line drawing, with color as the only selection cue. Such selection would be next to impossible if visual attention could only be applied to unparsed, unitary regions of visual space.

In Experiment 2, we found that the effects of common color on response competition can be strong enough to override those of proximity under some circumstances. This observation is analogous to the results of Driver and Baylis (1989) for common motion. The problem for the notion that visual attention is assigned to unparsed regions of the visual field, as implied by a literal version of the spotlight or zoom-lens metaphors, is particularly apparent in such cases, in which a nonspatial grouping factor overrides the effects of target-distractor separation. We must, however, place a caveat on the generality of these results. Klein (personal communication, 1989) and Kramer (personal communication, 1990) have separately conducted unpublished experiments following the same logic as those of Driver and Baylis (1989) or the present Experiment 2; that is, they set target-distractor grouping on a nonspatial factor against target-distractor separation and examined which factor would exert the greatest influence on response competition. In contrast to Driver and Baylis (1989), neither researcher found that common motion overrode proximity to produce a reverse distance effect, although effects of grouping by common motion were observed. In contrast with the present Experiment 2, Kramer (personal communication, 1990) did not find that common color fully reversed the proximity effect. Although full consideration of these data must await their publication, they clearly demonstrate that the phenomenon of nonspatial grouping's having stronger effects than those of proximity may not always occur. We will naturally be somewhat disappointed if the reverse distance effects that we have observed do not obtain for a wide range of parameters, but this outcome is not entirely surprising. The design of these experiments sets one factor (e.g., color) against another (e.g., position), and which factor dominates will presumably depend upon the exact parameters chosen for each factor. This point, which was noted by Wertheimer (1923, 
p. 313), is demonstrated by the visual search data on the relative importance of color and proximity discussed earlier.

The present Experiment 2 and the studies of Driver and Baylis (1989) do not demonstrate that common color or motion will always override the effects of proximity on response competition, but simply show that this outcome is possible even though it is not to be expected given certain theoretical accounts of visual attention. Nevertheless, it will clearly be informative to identify what the boundary conditions are for reverse distance effects to be observed. Kramer (personal communication, 1990) has identified one factor that may be important. He has found stronger effects of nonspatial grouping on response competition in a forcedpaced procedure than he has found when subjects initiate each trial. He suggests that the relative influence of targetdistractor proximity and nonspatial grouping may depend on the time that is available to subjects for focusing their attention on a particular location prior to display onset. If so, the implication would be that spatial mechanisms of selection can modulate the effects of nonspatial grouping in a top-down manner. This would be an interesting elaboration of our view, but not a falsification. We do not claim that mechanisms of visual attention cannot be directed spatially; there is clear evidence that they can (see, e.g., Posner, 1980). Rather, we wish to emphasize that location is not the sole medium for visual attention.

Our findings that color (Experiment 1) and good continuation (Experiments 5 and 6) affect the amount of response competition observed at a constant target-distractor separation may be less controversial than the disputed reverse distance effects. However, they also pose a problem for literal versions of the spotlight or zoom-lens metaphors, by demonstrating that the interference observed from a distractor is not solely a function of its distance from the known target location. Although the metaphors might be elaborated to accommodate these data by suggesting that the spotlight or zoom lens adopts the configuration of the group, the metaphors would thereby become diluted. Spotlights and zoom lenses do not adopt the configuration of the objects on which they are focused. Perhaps we take these metaphors too literally. Nevertheless, once it is suggested that the attentional spotlight or zoom lens is applied to perceptual groups, even though this is detrimental to performance because distractors are included within the focus, the central point of the grouping account of visual attention seems to have been accepted.

The present data demonstrate unequivocally that the amount of response competition produced by incongruent distractors is not solely a function of their distance from the target, even in static displays in which the target location is known in advance. Response competition also depends on whether target and distractor share a common color, and on whether they are grouped by good continuation. From a methodological point of view, our discussion of the conflicting data on whether nonspatial grouping factors can override proximity suggests that whether further nonspatial grouping factors exert any influence on response competition may be elucidated most clearly if their effect is examined at constant target-distractor separations. One further methodological point can be made. Some of our present findings (i.e., the comparison between Experiments 2 and 3, and between Experiments 7 and 8) imply that target-distractor grouping is a competitive process. Which distractor is most strongly linked to the target within any display may be as important as the absolute level of target-distractor grouping in that display. If so, within-display manipulations of target-distractor grouping are likely to provide a more sensitive measure of any grouping effects than are between-display manipulations.

\section{REFERENCES}

Bouma, H. (1970). Interaction effects in parafoveal letter recognition. Nature, 226, 177-178.

BrondBent, D. E. (1982). Task combination and selective intake of information. Acta Psychologica, 50, 253-290.

Driver, J., BAylis, G. C. (1989). Movement and visual attention: The spotlight metaphor breaks down. Journal of Experimental Psychology: Human Perception \& Performance, 15, 448-456.

Driver, J., BAyLIS, G. C. (1991). Target-distractor separation and feature integration in visual attention to letters. Acta Psychologica, 76, 101-119.

DUNCAN, J. (1984). Selective attention and the organization of visual information. Journal of Experimental Psychology: General, 113, 501-517.

Duncan, J., \& Humphreys, G. W. (1989). Visual search and stimulus similarity. Psychological Review, 96, 433-458.

ErIKsen, B. A., ERIKSEN, C. W. (1974). Effects of noise letters upon the identification of a target letter in a nonsearch task. Perception \& Psychophysics, 16, 143-149.

ERIKSEN, C. W., \& ST. JAMES, J. D. (1986). Visual attention within and around the field of focal attention: A zoom lens model. Perception \& Psychophysics, 40, 225-240.

ERIKSEN, C. W., SChUltz, D. W. (1979). Information processing in visual search: A continuous flow conception and experimental results. Perception \& Psychophysics, 25, 249-263.

Hagenaar, R., Van der Heuden, A. H. C. (1986). Target-noise separation in visual selective attention. Acta Psychologica, 62, 161-176.

Harms, L., \&undesen, C. (1983). Color segregation and selective attention in a nonsearch task. Perception \& Psychophysics, 33, 11-19.

HUMPHREYs, G. W. (1981). Flexibility of attention between stimulus dimensions. Perception \& Psychophysics, 30, 291-302.

HumphreYs, G. W., \& BrUCE, V. (1989). Visual cognition: Computational, experimental and neuropsychological perspectives. Hillsdale, NJ: Erlbaum.

Kahneman, D., Henik, A. (1981). Perceptual organization and attention. In M. Kubovy \& J. R. Pomerantz (Eds.), Perceptual organization (pp. 181-211). Hillsdale, NJ: Erlbaum.

LABERGE, D. (1983). Spatial extent of attention to letters and words. Journal of Experimental Psychology: Human Perception \& Performance, 9, 371-380.

MARR, D. (1982). Vision: A computational investigation into the human representation and processing of visual information. San Francisco: W. H. Freeman.

Merikie, P. M., Coltheart, M., Lowe, D. G. (1971). On the selective effects of a patterned masking stimulus. Canadian Joumal of Psychology, 25, 264-279.

Miller, J. (1991). The flanker compatibility effect as a function of visual angle, attentional focus, visual transients, and percepual load: A search for boundary conditions. Perception \& Psychophysics, 49, 270-288.

O'HARA, W., ERIKSEN, C. W. (1979). Word unitization examined using an interference paradigm. Bulletin of the Psychonomic Society, 14, 81-84. 
Posner, M. I. (1980). Orienting of attention. Quarterly Joumal of Experimental Psychology, 32, 3-26.

Prinzmetal, W. (1981). Principles of feature integration in visual perception. Perception \& Psychóphysics, 30, 330-340.

RABBrTt, P. M. A. (1966). Errors and error correction in choice response tasks. Journal of Experimental Psychology, 71, 264-272.

Rock, I., GutmaN, D. (1981). Effect of inattention on form perception. Journal of Experimental Psychology: Human Perception \& Performance, 7, 275-285.

TIPPER, S. P. (1985). The negative priming effect: Inhibitory effects of ignored primes. Quarterly Journal of Experimental Psychology, 37A, 571-590.

Treisman, A. [M.] (1982). Perceptual grouping and attention in visual search for features and for objects. Journal of Experimental Psychology: Human Perception \& Performance, 8, 194-214.

Treisman, A. [M.] (1988). Features and objects: The Fourteenth Bartlett Memorial Lecture. Quarterly Journal of Experimental Psychology, 40A, 201-237.

Treisman, A. M., Gelade, G. (1980). A feature-integration theory of attention. Cognitive Psychology, 12, 97-136.

TSAL, Y. (1983). Movements of attention across the visual field. Jour- nal of Experimental Psychology: Human Perception \& Performance, 9, 523-530.

TsAL, Y., LAvIE, N. (1988). Attending to color and shape: The special role of location in selective visual processing. Perception \& Psychophysics, 44, 15-21.

WERTHEIMER, M. (1922). Untersuchungen zur Lehre von der Gestalt I. Psychologische Forschung, 1, 48-57.

WERTHEIMER, M. (1923). Untersuchungen zur Lehre von der Gestalt II. Psychologische Forschung, 4, 301-350.

WILKINSON, J. (1986). SYSTAT, the system for statistics. Evanston, IL: Illinois University Press.

Wolfe, J. M., CAve, K. R., \& Franzel, S. L. (1989). Guided search: An alternative to the feature integration model for visual search. Journal of Experimental Psychology: Human Perception \& Performance, $15,419-433$.

YEH, Y.-Y., ERIKSEN, C. W. (1984). Name codes and features in the discrimination of letter forms. Perception \& Psychophysics, 36, 225-233.

(Manuscript received August 24, 1990; revision accepted for publication August 5, 1991.) 